\section{OPEN ACCESS}

Edited and reviewed by:

Ying Zhang,

Zhejiang University, China

*Correspondence:

X. Frank Yang

xfyang@iu.edu

Yongliang Lou

Iy@wmu.edu.cn

Specialty section:

This article was submitted to Infectious Diseases - Surveillance,

Prevention and Treatment,

a section of the journal

Frontiers in Medicine

Received: 17 February 2021 Accepted: 01 March 2021

Published: 31 March 2021

Citation:

Liu Q, Xu H, Zhang Y, Yang J, Du J,

Zhou Y, Yang XF and Lou Y (2021)

Corrigendum: Role of HK2 in the Enzootic Cycle of Borrelia burgdorferi. Front. Med. 8:668709.

doi: $10.3389 /$ fmed.2021.668709

\title{
Corrigendum: Role of HK2 in the Enzootic Cycle of Borrelia burgdorferi
}

\author{
Qiang Liu ${ }^{1,2}$, Haijun $\mathrm{Xu}^{3}$, Yan Zhang ${ }^{1,2,4}$, Jing Yang ${ }^{1,2}$, Jimei $\mathrm{Du}{ }^{1}$, Yan Zhou ${ }^{1}$, \\ $X$. Frank Yang ${ }^{2 *}$ and Yongliang Lou ${ }^{1 *}$
}

' Wenzhou Key Laboratory of Sanitary Microbiology, Key Laboratory of Laboratory Medicine, Ministry of Education, School of Laboratory Medicine and Life Sciences, Wenzhou Medical University, Wenzhou, China, ${ }^{2}$ Department of Microbiology and Immunology, Indiana University School of Medicine, Indianapolis, IN, United States, ${ }^{3}$ State Key Laboratory of Rice Biology and Ministry of Agriculture Key Laboratory of Agricultural Entomology, Institute of Insect Sciences, Zhejiang University, Hangzhou, China, ${ }^{4}$ Optometry and Eye Hospital and School of Ophthalmology, School of Biomedical Engineering, Wenzhou Medical University, Wenzhou, China

Keywords: lyme disease, Borrelia (Borreliella) burgdorferi, two-component system (TCS), Rrp2-HK2, OspC

\section{A Corrigendum on}

Role of HK2 in the Enzootic Cycle of Borrelia burgdorferi

by Liu, Q., Xu, H., Zhang, Y., Yang, J., Du, J., Zhou, Y., et al. (2020). Front. Med. 7:573648. doi: $10.3389 /$ fmed.2020.573648

In the original article, there was a mistake in Figure 5 as published. Due to an error in compiling multi-panel images, a gap between the image of "B31-A3" and the image of "B31A3/flaBp-HD-GYP; B31A3/flaBp-hk2" was omitted. In this figure, unphosphorylated Rrp2 (lower lane) serves as an internal control for each sample. Overproduction of an unrelated protein HD-GYP (B31A3/flaBp-HD-GYP) serves as the negative control for overproduction of Hk2 (B31A3/flaBp-hk2), showing a reduction of Rrp2 phosphorylation by overexpression of Hk2. The corrected Figure 5 appears below.

The authors apologize for this error and state that this does not change the scientific conclusions of the article in any way. The original article has been updated.

Copyright $\odot 2021$ Liu, Xu, Zhang, Yang, Du, Zhou, Yang and Lou. This is an open-access article distributed under the terms of the Creative Commons Attribution License (CC BY). The use, distribution or reproduction in other forums is permitted, provided the original author(s) and the copyright owner(s) are credited and that the original publication in this journal is cited, in accordance with accepted academic practice. No use, distribution or reproduction is permitted which does not comply with these terms. 


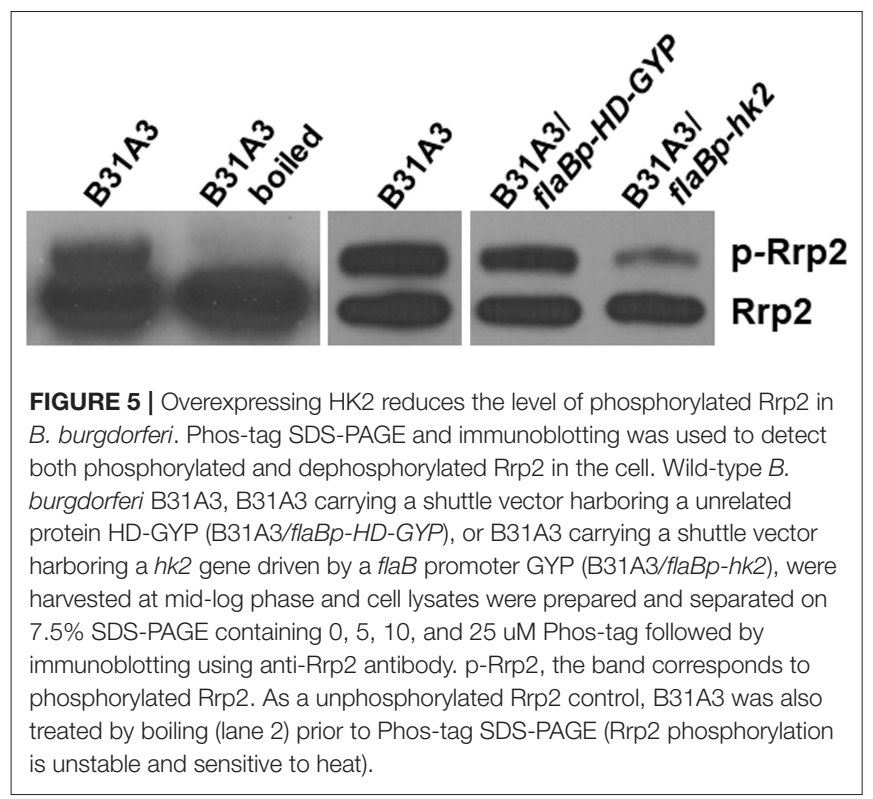

